\title{
Presence of Autoantibodies in Erosive Hand Osteoarthritis and Association with Clinical Presentation
}

\author{
Myrthe Alexandra Maria van Delft, Sjoerd van Beest ${ }^{(1)}$, Margreet Kloppenburg, \\ Leendert Adrianus Trouw, and Andreea Ioan-Facsinay
}

\begin{abstract}
Objective. To investigate whether 3 rheumatoid arthritis-associated antibodies [rheumatoid factor (RF) and anticitrullinated protein antibodies (ACPA) or anticarbamylated protein (anti-CarP) antibodies] are present in hand osteoarthritis (HOA) and associate with erosive OA (EOA).

Methods. Anti-CarP IgG was measured by ELISA in baseline sera of patients with HOA from 3 cohorts: HOSTAS $(n=510,27.2 \% \operatorname{EOA}), \operatorname{ECHO}(n=47)$, and EHOA $(n=23)$, and in sera of healthy controls (HC; $\mathrm{n}=196$, mean age $44.1 \mathrm{yrs}, 51.0 \%$ women). Moreover, ACPA-IgG and RF-IgM were additionally determined in HOSTAS and HC. The prevalence of autoantibodies was compared between HOA and $\mathrm{HC}$ and between erosive and nonerosive HOA. In HOSTAS, hand radiographs were scored (Kellgren-Lawrence, Osteoarthritis Research Society International osteophyte and joint space narrowing) and C-reactive protein (CRP) levels, representing inflammation, were assessed. Groups were compared using nonparametric tests.

Results. The prevalence of anti-CarP was low and not significantly different between the total HOA group and HC $(6.6 \%$ vs $3.6 \%, \mathrm{p}=0.12)$. In HOSTAS, the prevalence of all tested autoantibodies was low (anti-CarP 7.1\%, ACPA 0.8\%, RF 6.1\%), and there were no significant differences observed between HOA patients and $\mathrm{HC}$ or between erosive and nonerosive HOA. Further, radiographic damage and CRP levels were similar in anti-CarP+ and anti-CarP-, and RF+ and RF- HOSTAS patients.

Conclusion. The prevalence of autoantibodies is similar in HOA patients and HC, and these autoantibodies are not associated with erosive disease, structural damage, or inflammation in patients with HOA, indicating that another mechanism is driving erosive disease. (First Release September 15 2018; J Rheumatol 2019;46:101-5; doi:10.3899/jrheum.180256)
\end{abstract}

Key Indexing Terms: OSTEOARTHRITIS EROSIVE HAND OSTEOARTHRITIS ANTICITRULLINATED PROTEIN ANTIBODIES ANTICARBAMYLATED PROTEIN ANTIBODIES

AUTOANTIBODIES RHEUMATOID FACTOR
From the Department of Rheumatology, Department of Clinical Epidemiology, and Department of Immunohematology and Blood Transfusion, Leiden University Medical Center, Leiden, the Netherlands.

Supported by the Dutch Arthritis Foundation (14-2-402 and LLP-24), the Innovative Medicines Initiative Joint Undertaking (IMI JU)-funded project BeTheCure (115142-2) and the IMI JU-funded project under grant agreement number 115770, resources of which are composed of financial contributions from the European Union's Seventh Framework Programme (FP7/2007-2013) and the European Federation of Pharmaceutical Industries and Associations (EFPIA) companies' in-kind contribution. See www.imi.europa.eu and www.approachproject.eu. This communication reflects the views of the authors and neither IMI nor the European Union and EFPIA are liable for any use that may be made of the information contained herein. L.A. Trouw is supported by a ZON-MW Vidi grant (91712334). L.A. Trouw is listed as an inventor in a patent application regarding the detection of anticarbamylated protein antibodies for rheumatoid arthritis.

M.A. van Delft, MSc, PhD student, Department of Rheumatology, Leiden University Medical Center; S. van Beest, $M D$, PhD student, Department of Rheumatology, Leiden University Medical Center; M. Kloppenburg, Professor, MD, PhD, Department of Rheumatology, Leiden University Medical Center, and Department of Clinical Epidemiology, Leiden University Medical Center; L.A. Trouw, Associate Professor, PhD, Department of Rheumatology, Leiden University Medical Center, and Department of Immunohematology and Blood Transfusion, Leiden
University Medical Center; A. Ioan-Facsinay, Assistant Professor, PhD, Department of Rheumatology, Leiden University Medical Center. M.A. van Delft and S. van Beest contributed equally to this work.

Address correspondence to Dr. A. Ioan-Facsinay, Department of Rheumatology, Leiden University Medical Center, P.O. Box 9600, 2300 RC Leiden, the Netherlands.E-mail: a.ioan@lumc.nl

Accepted for publication July 5, 2018.

Osteoarthritis (OA) is a joint disease characterized by cartilage damage [joint space narrowing (JSN)], bone abnormalities (osteophytes, bone marrow lesions), and inflammation $^{1}$. One of its most prevalent clinical phenotypes is hand OA (HOA), which can evolve to erosive OA (EOA) ${ }^{1}$. In EOA, central erosions and subchondral destruction are seen, and imaging studies have indicated that in EOA, hand joints display more inflammatory signs than in nonerosive $\mathrm{HOA}^{2}$. Because patients with EOA have a higher clinical burden than nonerosive HOA, it is important to understand the mechanisms involved in development of EOA to develop therapeutic interventions.

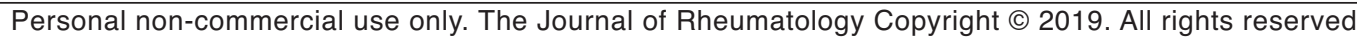


Autoantibodies directed against posttranslationally modified proteins, such as anticitrullinated protein antibodies (ACPA) and anticarbamylated protein (anti-CarP) antibodies, are a hallmark of rheumatoid arthritis (RA) ${ }^{3,4}$. Together with rheumatoid factors (RF), these antibodies can be detected in sera many years before the disease becomes clinically manifest ${ }^{3}$. In patients with RA, the presence of anti-CarP antibodies and ACPA is associated with more severe joint damage and inflammation ${ }^{3,5}$. ACPA and anti-CarP antibodies are mostly present in patients with RA but can also be detected in other clinical conditions ${ }^{6,7}$. Interestingly, these antibodies are present in a small subset of patients with systemic lupus erythematosus (SLE) as well, in which the antibodies associate with bone erosions ${ }^{8}$. This suggests the possible involvement of autoantibodies in inflammation and joint damage in conditions other than RA. Therefore, we hypothesized that autoantibodies are present in HOA and associate with erosive disease.

\section{MATERIALS AND METHODS}

Patients and controls. In this study, 196 healthy controls (HC) and 580 primary HOA patients from 3 different cohorts were included. Consecutive HOA patients visiting the Leiden University Medical Center (LUMC) rheumatology outpatient clinic were included in the HOSTAS $(n=510)$ and ECHO $(n=47)$ cohorts. The remaining patients $(n=23)$ were LUMC participants in the erosive HOA (EHOA) multicenter study9,10,11 (Table 1). The patients with OA were included between May 2008 and October 2015. Inclusion and exclusion criteria differed slightly between the cohorts and were described in detail previously ${ }^{9,10,11}$. Briefly, patients could participate when primary HOA was diagnosed according to the clinical judgment of the treating rheumatologist, in the absence of any other pathological condition that could explain the patient's symptoms (including inflammatory rheumatic diseases). In addition, ECHO and EHOA participants had to meet the American College of Rheumatology criteria for HOA, and were excluded if they previously tested RF- or ACPA-positive. Patients with EHOA also had to have at least 1 (pre-) erosive interphalangeal (IP) joint on conventional radiography. Baseline serum samples from all patients were analyzed. HC samples were acquired from persons living in the Leiden region, as described previously ${ }^{12}$. These persons were healthy and without any kind of rheumatic disease. Informed consent was obtained for all individuals and all protocols were approved by the medical ethical committee of the LUMC (EHOA: EudraCT Number 2007-003994-18, HOSTAS: CCMO reference NL26201.058.08, ECHO: CCMO reference NL17809.058.07).

Laboratory analysis of anti-CarP and ACPA-IgG, RF-IgM and C-reactive protein $(C R P)$. In-house ELISA were used to detect anti-CarP IgG, ACPA-IgG, and RF-IgM ${ }^{12}$. Protein carbamylation was performed and verified as described previously ${ }^{12}$. Briefly, plates coated with $10 \mu \mathrm{g} / \mathrm{ml}$ carbamylated fetal calf serum (CaFCS, Bodinco) or non-modified FCS
(Bodinco) were blocked with phosphate buffered saline (PBS)/1\% bovine serum albumin (BSA) and subsequently used to detect anti-CarP IgG antibodies in 50 -times diluted sera, upon overnight incubation at $4^{\circ} \mathrm{C}$. For detection of ACPA-IgG, plates were coated with $1 \mu \mathrm{g} / \mathrm{ml}$ cyclic citrullinated peptide (CCP) 2-citrulline or CCP2-arginine (produced and provided by $\mathrm{Dr}$. J.W. Drijfhout, Immunohematology and Blood Transfusions, LUMC), for RF-IgM, plates were coated with $10 \mu \mathrm{g} / \mathrm{ml}$ human IgG (Jackson immune research, 009-000-003) and blocked with PBS/1\% BSA. Sera, diluted 50 times for ACPA and 100 times for RF-IgM, were incubated for $1 \mathrm{~h}$ at $37^{\circ} \mathrm{C}$. Bound human IgG or IgM were detected using matched horseradish peroxidase (HRP)-conjugated secondary antibodies [rabbit antihuman IgG/HRP (DAKO P0214) or goat antihuman IgM/HRP (Invitrogen, 627520)]. ABTS (2,2'-azino-bis 3-ethylbenzothiazoline-6-sulphonic acid, Sigma Aldrich, A1888) was added to visualize the HRP enzyme activity and absorbance at $415 \mathrm{~nm}$ was measured. Levels higher than the mean plus 2-times the SD (M $+2 \mathrm{SD}$ ) of the specific anti-CarP or RF levels in HC were considered positive. The specific anti-CarP response was calculated by subtracting the response of non-modified FCS from the response of CaFCS. For ACPA-IgG, the commercially advised cutoff of 25 units was used. Only sera positive for CCP2-citrulline and negative for CCP2-arginine were considered positive.

CRP levels were measured by the Roche Modular P800 (Diamond Diagnostics Inc.) at the routine diagnostic laboratory of the LUMC.

Radiographs. In HOSTAS, conventional dorsopalmar radiographs of both hands were obtained. The proximal and distal IP joints were scored according to Verbruggen-Veys (VV) anatomical phases (N-S-J-E-R ${ }^{13}$. Patients with at least 1 joint in the $\mathrm{E}$ phase (erosive) or $\mathrm{R}$ phase (remodeled) were considered to have EOA.

All IP joints, metacarpophalangeal joints, and first carpometacarpal (CMC-1) joints were scored following the Kellgren-Lawrence (KL) arthritis grading scale $(0-4 \text {, maximum sum score } 120)^{13}$. In addition, IP joints, CMC-1 joints, and scaphotrapeziotrapezoid (STT) joints were scored for osteophytes and JSN separately using the Osteoarthritis Research Society International (OARSI) atlas (first IP and STT 0-1, other joints 0-3, maximum sum score $58)^{13}$. Scoring was performed by 1 well-trained reader, blinded for clinical and demographic data. Intrareader reliability was assessed on a randomly selected sample $(n=31)$ and resulted in high ICC (EOA status 0.92, sum scores: KL 0.95, OARSI osteophytes 0.97, OARSI JSN 0.93).

Statistical analysis. Statistical analyses were performed using SPSS version 23 (IBM). Mann-Whitney U tests were carried out to determine differences in anti-CarP antibody levels between total HOA patients and $\mathrm{HC}$, differences in any antibody levels between HOSTAS and HC, and between erosive and nonerosive OA patients within HOSTAS. Moreover, the same statistical test was used to determine differences in radiographic features (KL score, OARSI osteophyte, and JSN scores) between anti-CarP IgG or RF-IgM-positive and -negative HOSTAS patients. Further, the Pearson's chi-square test was used to determine whether there was a difference in the number of anti-CarP IgG-, ACPA-IgG-, and RF-IgM-positive subjects between the same groups as described for the antibody levels comparison. The same test was carried out to investigate whether there was a difference in the number of HOSTAS patients with a raised CRP level $(>5 \mathrm{mg} / \mathrm{ml})$ between anti-CarP IgG- or RF-IgM-positive and -negative patients. P values $<0.05$ were considered statistically significant.

Table 1. Baseline characteristics of cohorts.

\begin{tabular}{lcccc}
\hline Patient Characteristics & HOSTAS, $\mathrm{n}=510$ & EHOA, $\mathrm{n}=23$ & ECHO, $\mathrm{n}=47$ & Healthy Controls, $\mathrm{n}=196$ \\
\hline Age, $\mathrm{yrs}$, mean \pm SD & $61.0 \pm 8.6$ & $57.1 \pm 7.0$ & $63.4 \pm 9.2$ & $44.1 \pm 13.9$ \\
Women, $\%$ & 85.7 & 73.9 & 89.4 & 51.0 \\
BMI, $\mathrm{kg} / \mathrm{m}^{2}$, mean \pm SD & $27.1 \pm 4.7, \mathrm{n}=496$ & $26.3 \pm 4.7$ & $27.4 \pm 4.5, \mathrm{n}=45$ & $23.7 \pm 3.0, \mathrm{n}=195$ \\
Ever smoker, $\%$ (former/current) & $64.9(323 / 498)$ & 65.2 & $33.3(15 / 45)$ & $34.9(68 / 195)$ \\
Fulfilling ACR criteria ${ }^{a}, \%$ & 90.0 & 100 & 100 & - \\
\hline
\end{tabular}

${ }^{a}$ American College of Rheumatology classification criteria for hand OA. BMI: body mass index; OA: osteoarthritis; EHOA: erosive hand OA. 


\section{RESULTS}

Anti-CarP IgG and RF-IgM have a low prevalence in patients with $O A$. Anti-CarP IgG, ACPA-IgG, and RF-IgM were measured. Prevalences of 3.6\% for anti-CarP IgG and $4.1 \%$ for RF-IgM were observed in the control population, using $\mathrm{M}+2 \mathrm{SD}$ in $\mathrm{HC}$ as cutoff. The prevalence of ACPA-IgG was $1.5 \%$ in $\mathrm{HC}$, using the commercially available cutoff. Applying the same methods, a prevalence of $6.6 \%$ was observed for anti-CarP IgG in the total HOA population; this was not significantly different from the control population $(\mathrm{p}=0.12)$. The level of anti-CarP IgG was also not different between the total HOA group and $\mathrm{HC}(\mathrm{p}=0.37)$. In HOSTAS, a prevalence of $7.1 \%$ was observed for anti-CarP $\mathrm{IgG}, 0.8 \%$ for ACPA IgG, and $6.1 \%$ for RF-IgM. These prevalences were not significantly different compared to HC (anti-CarP $\mathrm{p}=0.08$; ACPA $\mathrm{p}=0.37$; RF $\mathrm{p}=0.30$ ). Further, for the most prevalent autoantibodies, anti-CarP IgG and RF-IgM, antibody levels did not differ between these groups (anti-CarP p $=0.67$; RF p $=0.82$ ). Dot plots for anti-CarP $\mathrm{IgG}$ in $\mathrm{HC}$ and total HOA are depicted in Figure 1A. All tested autoantibodies in HOSTAS are depicted in Figure 1B.
Although previously known RF-IgM or ACPA IgG seropositivity was an exclusion criterion in the EHOA and ECHO cohorts, it was not mandatory to test for these antibodies; this explains why we found 2 of these patients to be RF-IgM-positive (1 EHOA and $1 \mathrm{ECHO}$ ); however, all were ACPA-negative (data not shown).

In HOSTAS, 3 patients developed RA during a followup time of 2.0-8.4 years: 1 RF-IgM-positive patient was diagnosed with RF+/ACPA- RA after 28 months followup, and 2 triple-antibody-negative patients developed $\mathrm{RF}+/ \mathrm{ACPA}-$ and RF+/ACPA+ RA after 46 and 61 months followup time, respectively. The antibody levels, and clinical and demographic features were not significantly different for the patients who developed RA compared to the remainder of the HOSTAS cohort (data not shown).

Further, only 4 patients (all HOSTAS) were positive for $>1$ autoantibody: 2 for anti-CarP and ACPA, 1 for anti-CarP and RF, and 1 for ACPA and RF; none developed RA during a followup time of 3.4-6.1 years. There was 1 anti-CarP-positive patient (out of 36 positive) and 1 ACPA-positive patient (out of 4 positive) with increased

A.

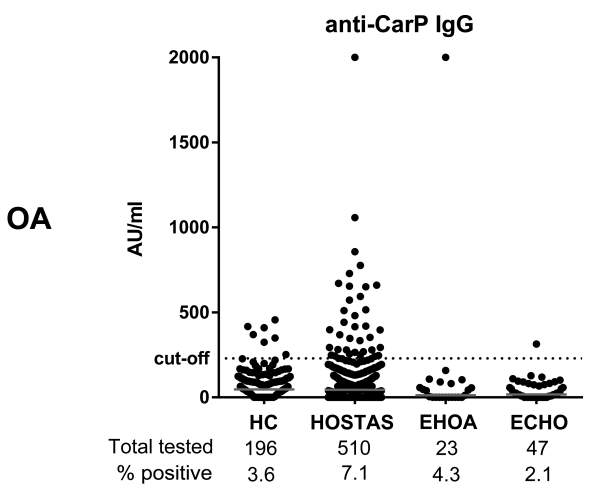

B.
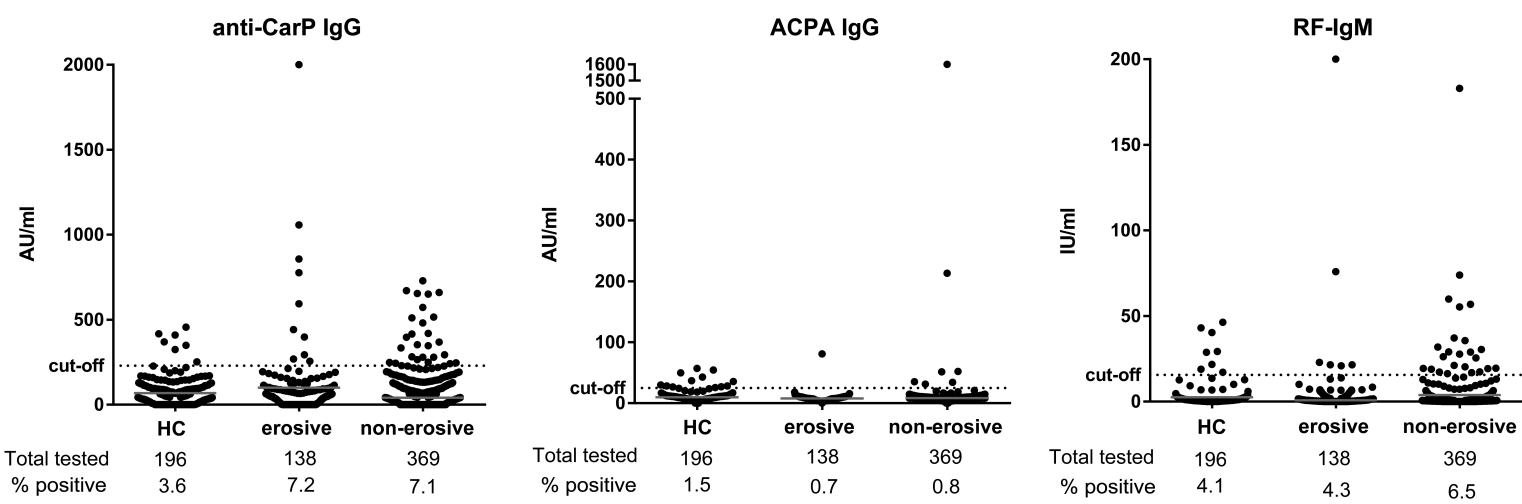

Figure 1. A. Autoantibodies in patients with HOA of HOSTAS, EHOA, and ECHO cohorts, and clinical features of HOSTAS. ELISA were performed to detect anti-CarP antibody, ACPA, and RF in sera of $196 \mathrm{HC}$ and 580 HOA patients (510 HOSTAS, 23 EHOA, and 47 ECHO). B. The HOSTAS group was subdivided in erosive and nonerosive HOA. The mean plus 2-times the SD in HC was established as the cutoff for the anti-CarP antibody and RF. For ACPA, a cutoff of $25 \mathrm{AU} / \mathrm{ml}$ was used. The dotted line represents the cutoff. The number of samples tested in each group and the prevalence, corrected for the response on nonmodified FCS or CCP2-arginine, is shown below the graphs. Mann-Whitney U test for level and Pearson's chi-square test for prevalence were performed in erosive versus nonerosive OA. HOA: hand osteoarthritis; EHOA: erosive HOA; anti-CarP: anticarbamylated protein antibody, ACPA: anticitrullinated protein antibodies, RF: rheumatoid factor, HC: healthy controls; AU: arbitrary units; FCS: fetal calf serum; CCP: cyclic citrullinated peptide.

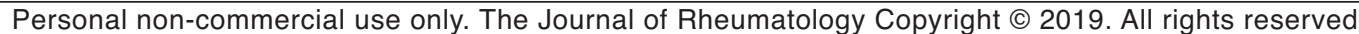


CRP, in contrast to none of the outliers (anti-CarP 2000 AU/ml, ACPA 1600 AU/ml, RF-IgM $200 \mathrm{IU} / \mathrm{ml}$ ) in all patients with HOA. Moreover, these patients did not differ from other HOA patients in demographic features or radiographic damage (data not shown).

Anti-CarP IgG and RF-IgM are not associated with erosive disease in $O A$. To investigate whether the presence of autoantibodies was different in EOA compared to nonerosive HOA, the largest available cohort, HOSTAS, was used, in which $27.2 \%$ of the patients $(n=138)$ had EOA. Neither the frequency (anti-CarP IgG p $=0.94$, RF-IgM p $=0.36$ ) nor the levels (anti-CarP IgG p = 0.69, RF-IgM p = 0.53) of the most prevalent autoantibodies were increased in EOA patients compared to nonerosive HOA (Figure 1B). To explore the possibility that using $\mathrm{VV}$ anatomic $\mathrm{E}$ and $\mathrm{R}$ phases as cutoff for EOA is too conservative, causing patients with early erosive disease to be miscategorized, we performed the same analyses with the preceding $\mathrm{J}$ phase in at least 1 joint as cutoff for EOA. This more sensitive approach yielded similar results (data not shown).

Further, no associations were found between the presence of anti-CarP or RF-IgM and increased radiographic damage or inflammation (i.e., CRP level > $5 \mathrm{mg} / \mathrm{l}$; Table 2).

\section{DISCUSSION}

In our study, we analyzed the presence of anti-CarP IgG, ACPA-IgG, and RF-IgM in patients with HOA. To our knowledge, this is the first time that autoantibodies were measured in such a large group of patients with OA. The results indicate that few HOA patients are positive for autoantibodies and these autoantibodies are not linked to erosive disease or inflammation in OA, in contrast to other inflammatory diseases in which bone erosions are present, such as RA and $\mathrm{SLE}^{3,8}$. We confirmed earlier results showing limited prevalence of ACPA in $\mathrm{HOA}^{14}$. Moreover, we detect prevalence of RF-IgM in HOA similar to that in a previous study ${ }^{15}$. Although this was a small study without detectable differences in RF-IgM between EOA and nonerosive OA, we do not observe any association of RF-IgM with EOA.

Myeloperoxidase (MPO) is one of the enzymes involved in carbamylation and the levels of MPO are increased during inflammation ${ }^{3}$. Interestingly, patients with HOA do have higher serum levels of MPO than $\mathrm{HC}^{16}$. Moreover, EOA have even higher serum levels of MPO compared to nonerosive EOA, which is in line with the presence of more inflammatory signs in EOA compared to nonerosive $\mathrm{HOA}^{2,16}$, as MPO is increased during inflammation. Similarly, patients with RA and SLE also have increased serum MPO levels compared to $\mathrm{HC}^{17}$, and RA patients with more inflammatory signs have higher levels of MPO than patients with less inflammatory RA. Although it is currently unknown whether the inflammation-associated increased levels of serum MPO are associated with increased carbamylation of proteins (in joints), it is conceivable that increased MPO levels can lead to more carbamylation and potentially reduce tolerance toward carbamylated proteins. Previous studies analyzed the presence of anti-CarP antibodies in sera of patients with conditions that are known to have an increased degree of carbamylation due to the following: increased levels of urea (renal failure), increased levels of thyocyanate (smoking), or chronic inflammation (inflammatory bowel disease) $)^{7}$. Compared to patients with RA, the percentage of anti-CarP positivity was much lower, and with the exception of renal failure, not different from that of $\mathrm{HC}^{7}$. Collectively, the current data and these previous studies indicate that increased protein carbamylation (e.g., through increased levels of MPO) is not sufficient to drive anti-CarP antibody production.

In a case report, carbamylated proteins were shown to be present in both erosive and nonerosive joints ${ }^{18}$ and our current study also indicates that carbamylated proteins are present in the joints of patients with OA. Carbamylated proteins can therefore be present in OA with no apparent breach of tolerance against these antigens. Whether this is due to the type of protein that is carbamylated, its localization, the degree of carbamylation, or other factors remains to be investigated in future studies.

A strength of our study is the replication of autoantibody measurements in 3 different cohorts. Because the HOSTAS cohort includes patients in all disease stages, from early to severe, we were able to also study the link between radiographic damage and antibody status.

A limitation of our study is that $\mathrm{HC}$ and patients with $\mathrm{OA}$ were not age- and sex-matched, because it is difficult to

Table 2. Other clinical features of HOSTAS cohort.

\begin{tabular}{|c|c|c|c|c|c|c|}
\hline Variables & anti-CarP+, n $=36$ & anti-CarP-, $\mathrm{n}=471$ & $\mathrm{p}$ & $\mathrm{RF}+, \mathrm{n}=30$ & $\mathrm{RF}-, \mathrm{n}=477$ & $\mathrm{p}$ \\
\hline Sum-score KL $(0-120)^{\mathrm{a}}$ & $17.5(9.5-30.75)$ & $18.0(8.0-29.0)$ & 0.74 & $13.5(8.75-30.5)$ & $18.0(8.0-30.5)$ & 0.59 \\
\hline Sum-score OARSI osteophytes $(0-58)^{\mathrm{a}}$ & $9.5(6.0-19.0)$ & $10.0(4.0-18.0)$ & 0.64 & $8.0(4.75-16.25)$ & $10.0(4.5-18.0)$ & 0.74 \\
\hline $\mathrm{CRP}$ raised $(>5.0 \mathrm{mg} / \mathrm{l})^{\mathrm{b}}, \%(\mathrm{n} /$ total $)$ & $11.5(3 / 26)$ & $10.4(34 / 326)$ & 0.86 & $7.7(2 / 26)$ & $10.7(35 / 326)$ & 0.63 \\
\hline
\end{tabular}

Values are median (IQR) unless otherwise specified. ${ }^{\text {a }}$ For 3 patients, no hand radiographs were available; Mann-Whitney U test was performed. ${ }^{\mathrm{b}}$ Only 352 patients had their CRP levels measured within 22 days from baseline; Pearson's chi-square test was performed. Anti-CarP: anticarbamylated protein antibodies; RF: rheumatoid factor; KL: Kellgren-Lawrence arthritis grading scale; OARSI: Osteoarthritis Research Society International; CRP: C-reactive protein; IQR: interquartile range.

Personal non-commercial use only. The Journal of Rheumatology Copyright $@$ 2019. All rights reserved 
assemble such a large group of $\mathrm{HC}$ around the age of 60 years, $80 \%$ women, and completely free of HOA. The HC group was used to set the cutoff for anti-CarP positivity and it was considered more important to use a control group that reflected the overall population of the area in which the disease population is living. Importantly, we did not observe a relationship between the presence of anti-CarP antibody in $\mathrm{HC}$ and sex, age, or smoking (data not shown).

In patients with HOA, just a small subset is positive for the RA-associated autoantibodies anti-CarP IgG, ACPA-IgG, or RF-IgM, and we did not observe an association between the presence of these autoantibodies with erosive disease or inflammation in OA. Altogether, the data suggest that another mechanism, possibly independent of autoantibodies, is driving erosive disease in HOA.

\section{ACKNOWLEDGMENT}

We thank J.C. Kwekkeboom for her help in collecting the samples, C. Kromme for his help with designing the data file, and W. Damman for her help in the scoring of the radiographs.

\section{REFERENCES}

1. Kloppenburg M, Kwok WY. Hand osteoarthritis - a heterogeneous disorder. Nat Rev Rheumatol 2011;8:22-31.

2. Kortekaas MC, Kwok WY, Reijnierse M, Huizinga TW, Kloppenburg M. In erosive hand osteoarthritis more inflammatory signs on ultrasound are found than in the rest of hand osteoarthritis. Ann Rheum Dis 2013;72:930-4.

3. Trouw LA, Rispens T, Toes RE. Beyond citrullination: Other post-translational protein modifications in rheumatoid arthritis. Nat Rev Rheumatol 2017;13:331-9.

4. Shi J, Knevel R, Suwannalai P, van der Linden MP, Janssen GM, van Veelen PA, et al. Autoantibodies recognizing carbamylated proteins are present in sera of patients with rheumatoid arthritis and predict joint damage. Proc Natl Acad Sci U S A 2011;108:17372-7.

5. Derksen VF, Ajeganova S, Trouw LA, van der Helm-van Mil AH, Hafstrom I, Huizinga TW, et al. Rheumatoid arthritis phenotype at presentation differs depending on the number of autoantibodies present. Ann Rheum Dis 2016;76:716-20.

6. Hermans MP, van der Velden D, Montero Cabezas JM, Putter H, Huizinga TW, Kuiper J, et al. Long-term mortality in patients with ST-segment elevation myocardial infarction is associated with anti-citrullinated protein antibodies. Int J Cardiol 2017;240:20-4.

7. Verheul MK, van Erp SJ, van der Woude D, Levarht EW, Mallat MJ, Verspaget HW, et al. Anti-carbamylated protein antibodies: a specific hallmark for rheumatoid arthritis. Comparison to conditions known for enhanced carbamylation; renal failure, smoking and chronic inflammation. Ann Rheum Dis 2016;75:1575-6.

8. Ziegelasch M, van Delft MA, Wallin P, Skogh T, Magro-Checa C, Steup-Beekman GM, et al. Antibodies against carbamylated proteins and cyclic citrullinated peptides in systemic lupus erythematosus: results from two well-defined european cohorts. Arthritis Res Ther 2016;18:289.

9. Damman W, Liu R, Kroon FPB, Reijnierse M, Huizinga TWJ, Rosendaal FR, et al. Do comorbidities play a role in hand osteoarthritis disease burden? Data from the Hand Osteoarthritis in Secondary Care cohort. J Rheumatol 2017;44:1659-66.

10. Kortekaas MC, Kwok WY, Reijnierse M, Huizinga TW, Kloppenburg M. Osteophytes and joint space narrowing are independently associated with pain in finger joints in hand osteoarthritis. Ann Rheum Dis 2011;70:1835-7.

11. Kloppenburg M, Ramonda R, Kwok WY, Bobacz K, Elewaut D, Frallonardo P, et al. OP0095 randomized, placebo-controlled trial to evaluate clinical efficacy and structure modifying properties of subcutaneous etanercept (ETN) in patients with erosive inflammatory hand osteoarthritis (OA) [abstract]. Ann Rheum Dis 2016;75 Suppl 2:90-1.

12. van Delft MAM, Verheul MK, Burgers LE, Derksen VFAM, van der Helm-van Mil AHM, van der Woude D, et al. The isotype and IgG subclass distribution of anti-carbamylated protein antibodies in rheumatoid arthritis patients. Arthritis Res Ther 2017;19:190.

13. Bijsterbosch J, Haugen IK, Malines C, Maheu E, Rosendaal FR, Watt I, et al. Reliability, sensitivity to change and feasibility of three radiographic scoring methods for hand osteoarthritis. Ann Rheum Dis 2011;70:1465-7.

14. Dolzani P, Assirelli E, Pulsatelli L, Addimanda O, Mancarella L, Peri G, et al. Systemic inflammation and antibodies to citrullinated peptides in hand osteoarthritis. Clin Exp Rheumatol 2011; 29:1006-9.

15. Vannini A, Fusconi M, Dall'Aglio AC, Tovoli F, Frisoni M, Zauli D. A seroimmunological profile of erosive hand osteoarthritis. Acta Reumatol Port 2013;38:39-43.

16. Punzi L, Ramonda R, Deberg M, Frallonardo P, Campana C, Musacchio E, et al. Coll2-1, coll2-1no2 and myeloperoxidase serum levels in erosive and non-erosive osteoarthritis of the hands. Osteoarthritis Cartilage 2012;20:557-61.

17. Wang W, Jian Z, Guo J, Ning X. Increased levels of serum myeloperoxidase in patients with active rheumatoid arthritis. Life Sci 2014;117:19-23.

18. Turunen S, Koivula MK, Melkko J, Alasaarela E, Lehenkari P, Risteli J. Different amounts of protein-bound citrulline and homocitrulline in foot joint tissues of a patient with anti-citrullinated protein antibody positive erosive rheumatoid arthritis. J Transl Med 2013;11:224. 